ÖSTERREICHISCHE AKADEMIE DER WISSENSCHAFTEN BALKAN-KOMMISSION

UNIVERSITÄT WIEN

INSTITUT FÜR SLAWISTIK

\title{
WIENER
}

\section{SLAVISTISCHES JAHRBUCH}

Band 57

Verlag der 


\title{
WIENER SLAVISTISCHES JAHRBUCH
}

Gedruckt mit Unterstützung der

Philologisch-Kulturwissenschaftlichen Fakultät der Universität Wien

\section{Herausgegeben}

von Seiten der Österreichischen Akademie der Wissenschaften: von Christian Hannick, Radoslav Katičić, Gerhard Neweklowsky

von Seiten des Instituts für Slawistik der Universität Wien:

von Juliane Besters-Dilger (Freiburg i. Br.), Vladimir Biti, Georg Holzer (Schriftleiter), Anna Kretschmer, Heinz Miklas, Stefan Michael Newerkla, Fedor B. Poljakov,

Stefan Simonek (Redakteur), Josef Vintr, Pavol Winczer, Alois Woldan

Administration und technische Bearbeitung: Sylvia Richter

\author{
Redaktionsadresse: \\ Institut für Slawistik \\ der Universität Wien \\ Universitätscampus AAKH, Hof 3, \\ Spitalgasse 2, 1090 Wien \\ Österreich - Austria \\ E-Mail: sylvia.richter@univie.ac.at
}

Die verwendete Papiersorte ist aus chlorfrei gebleichtem Zellstoff hergestellt, frei von säurebildenden Bestandteilen und alterungsbeständig.

Copyright (C) 2011 by Österreichische Akademie der Wissenschaften Wien

Alle Rechte vorbehalten

Druck: Druckerei Ferdinand Berger \& Söhne GesmbH, 3580 Horn

ISBN: 978-3-7001-7147-8

ISSN 0084-0041

http://hw.oeaw.ac.at/7147-8

http://verlag.oeaw.ac.at 